\title{
From multiplicative unitaries to quantum groups
}

\author{
S.L. Woronowicz* \\ Department of Mathematical Methods in Physics, \\ Faculty of Physics, University of Warsaw, \\ Hoża 74, 00-682 Warszawa, Poland
}

July 1995

\begin{abstract}
An alternative version of the theory of multiplicative unitaries is presented. Instead of the original regularity condition of Baaj and Skandalis we formulate another condition selecting manageable multiplicative unitaries. The manageability is the property of multiplicative unitaries coming from the quantum group theory. For manageable multiplicative unitaries we reproduce all the essential results of the original paper of Baaj and Skandalis and much more. In particular the existence of the antipode and its polar decomposition is shown.
\end{abstract}

\section{$0 \quad$ Introduction}

To explain the subject of the paper we consider the multiplicative unitary related to a locally compact group $G$. Let $H=L^{2}(G)$ be the Hilbert space of all measurable functions on $G$ square integrable with respect to the right - invariant Haar measure. One can easily verify that the formula

$$
(W x)\left(g, g^{\prime}\right)=x\left(g g^{\prime}, g^{\prime}\right)
$$

defines a unitary operator on $H \otimes H$. This operator often called Kac - Takesaki operator is very interesting. It contains the full information about the group $G$. This fact follows in the most transparent way from the famous Tatsuuma duality [6]. Let

$$
G \ni g \mapsto R_{g} \in B(H)
$$

be the right regular representation of $G$ : $\left(R_{g} x\right)\left(g^{\prime}\right)=x\left(g^{\prime} g\right)$ for any $x \in H$ and $g, g^{\prime} \in G$. Then for any non-zero $T \in B(H)$ we have

$$
\left\{W^{*}(I \otimes T) W=T \otimes T\right\} \Longleftrightarrow\left\{\begin{array}{c}
\text { There exists } g \in G \\
\text { such that } T=R_{g}
\end{array}\right\}
$$

\footnotetext{
*Supported by Komitet Badań Naukowych, grant No 2 P301 02007
} 
We list the most important properties of the Kac - Takesaki operator. First of all, it satisfies the following pentagonal equation:

$$
W_{23} W_{12}=W_{12} W_{13} W_{23} .
$$

In this formula, we use the leg numbering notation: $W_{k l}(k, l \in\{1,2,3\}, k<l)$ denotes the copy of $W$ acting on $H \otimes H \otimes H=L^{2}(G \times G \times G)$ affecting only the $k^{\text {th }}$ and $l^{\text {th }}$ variable:

$$
\begin{aligned}
& \left(W_{12} x\right)\left(g, g^{\prime}, g^{\prime \prime}\right)=x\left(g g^{\prime}, g^{\prime}, g^{\prime \prime}\right), \\
& \left(W_{13} x\right)\left(g, g^{\prime}, g^{\prime \prime}\right)=x\left(g g^{\prime \prime}, g^{\prime}, g^{\prime \prime}\right), \\
& \left(W_{23} x\right)\left(g, g^{\prime}, g^{\prime \prime}\right)=x\left(g, g^{\prime} g^{\prime \prime}, g^{\prime \prime}\right) .
\end{aligned}
$$

The verification of the pentagonal equation is a matter of a simple computations. One can easily check that

$$
\begin{aligned}
\left(W_{23} W_{12} x\right)\left(g, g^{\prime}, g^{\prime \prime}\right) & =x\left(g\left(g^{\prime} g^{\prime \prime}\right), g^{\prime} g^{\prime \prime}, g^{\prime \prime}\right), \\
\left(W_{12} W_{13} W_{23}\right)\left(g, g^{\prime}, g^{\prime \prime}\right) & =x\left(\left(g g^{\prime}\right) g^{\prime \prime}, g^{\prime} g^{\prime \prime}, g^{\prime \prime}\right)
\end{aligned}
$$

so, the pentagonal equation is equivalent to the associativity of the group rule.

Another interesting property is the regularity. To describe it, we consider the set $B(H)_{*}$ of all normal linear functionals defined on $B(H)$ and the flip $\Sigma \in B(H \otimes H)$ : $\Sigma(x \otimes y)=y \otimes x$ for all $x, y \in H$. In what follows, $L^{2}(H)$ will denote the space Hilbert space of all Hilbert-Schmidt class operators acting on $H$. It is known that $L^{2}(H)$ is canonically isomorphic to $L^{2}(G \times G)$ : an operator $T \in L^{2}(H)$ if and only if $T$ is an integral operator with the kernel belonging to $L^{2}(G \times G)$.

Let $x, y \in H$ and for any $a \in B(H), \omega(a)=(x \mid a y)$. Then $\omega \in B(H)_{*}$. One can easily check that $(\mathrm{id} \otimes \omega)(\Sigma W)$ is an integral operator with the square integrable kernel $K\left(g, g^{\prime}\right)=y(g) \bar{x}\left(g^{\prime} g^{-1}\right)$ and that the set of such kernels (when $x$ and $y$ run over $H$ ) is linearly dense in $L^{2}(G \times G)$. Therefore $(\mathrm{id} \otimes \omega)(\Sigma W) \in L^{2}(H)$ and the set of such operators is linearly dense in $L^{2}(H)$. Remembering that $L^{2}(H)$ is norm dense in the algebra $C B(H)$ of all compact operators acting on $H$ we get:

$$
\left\{(\mathrm{id} \otimes \omega)(\Sigma W): \omega \in B(H)_{*}\right\}^{\text {norm closure }}=C B(H) .
$$

In a fundamental and beautiful paper [3], Baaj and Skandalis presented an axiomatic approach to the theory of Kac - Takesaki operator. As basic axioms they used the unitarity of $W$, the pentagonal relation (0.1) and the regularity property (0.2). They were able to show that

$$
A=\left\{(\omega \otimes \mathrm{id}) W: \omega \in B(H)_{*}\right\}^{\text {norm closure }}
$$

and

$$
\hat{A}=\left\{(\mathrm{id} \otimes \omega) W^{*}: \omega \in B(H)_{*}\right\}^{\text {norm closure }}
$$


are $\mathrm{C}^{*}$-algebras and that $W \in M(\hat{A} \otimes A)$. In the special case considered above, $A=$ $C_{\infty}(G)$ and $\hat{A}=C^{*}(G)$.

Unfortunately the Baaj and Skandalis theory does not apply to all Kac - Takesaki operators related to quantum groups. It was discovered by Baaj $[1,2]$ that in general these operators are not regular. In the present paper we propose to replace the regularity by another property called manageability. To explain the sense of this concept we go back to the Kac - Takesaki operator $W$ related to a locally compact group. It has the following remarkable property: There exists a unitary operator $\widetilde{W}$ such that

$$
(x \otimes u|W| z \otimes y)=(\bar{z} \otimes u|\widetilde{W}| \bar{x} \otimes y)
$$

for all $x, y, z, u \in H$. Indeed one can easily check that the operator $\widetilde{W}=W^{*}$ satisfies (0.3). One can easily show that Kac - Takesaki operators related to quantum groups with involutive antipode have the same property.

The manageability is a natural generalization of the above property necessary to include into the theory the quantum groups with a nontrivial square of the antipode. The paper presents the theory of multiplicative unitaries based on three axioms: unitarity, pentagonal equation and manageability.

In the first section we introduce the notions of manageable multiplicative unitary and that of adapted unitary. The latter notion coincides with the concept of a unitary representation of the quantum group associated with a given manageable multiplicative unitary. We decided to change the terminology, because we had to consider the adapted operators before the corresponding quantum group is constructed. One should also notice that introducing adapted operators we do not assume explicitly that their 'second leg' is affiliated with the function $\mathrm{C}^{*}$-algebra of the group. This is what we are able to prove. Next we show that for any manageable multiplicative unitary $W$, its dual $\widehat{W}=\Sigma W^{*} \Sigma$ is manageable.

In the second part of Section 1 we formulate the theorems containing the main results of the paper. Theorem 1.5 deals with a manageable multiplicative unitary, Theorem 1.6 contains the corresponding results for adapted unitaries. The reader should notice that any multiplicative unitary is adapted to itself, so many Statements of Theorem 1.5 follows directly from the corresponding Statements of Theorem 1.6

In Section 2 we show that any unitary adapted to a manageable multiplicative unitary is manageable. Section 3 contain the proof that slicing an adapted unitary with respect to the second leg and taking the norm closure we obtain a $\mathrm{C}^{*}$-algebra. The same is shown for the first leg slicing. Then in Section 4 we show that the adapted operator belongs to the multiplier algebra of the of the tensor product of the two algebras. The most important point in the proofs is to show that the closure of the set

$$
\left\{(\operatorname{id} \otimes \omega)(\Sigma W): \omega \in B(H)_{*}\right\}
$$

contains sufficiently many one-dimensional operators. The essential difference between this paper and [3] lies in the different topology used for this purpose. Instead of the norm topology used by Baaj and Skandalis we use a Hilbert space topology on the space of modified Hilbert - Schmidt operators. 
Section 5 contains standard material on comultiplication. Section 6 is devoted to the scaling group: the analytical generator of the scaling group enters the polar decomposition of the antipode. The remaining factor of this decomposition (so called unitary antipode) is introduced and investigated in Section 7.

In this paper we freely use the $\mathrm{C}^{*}$-algebra notation described in our previous papers (e.g: [8]). In particular for any $\mathrm{C}^{*}$-algebras $A$ and $B, \operatorname{Mor}(A, B)$ denotes the set of all non-degenerate ${ }^{*}$-homomorphisms acting from $A$ into the multiplier algebra $M(B)$ of the algebra $B$.

\section{Basic definitions and results}

Let $H$ be a separable Hilbert space and $\bar{H}$ be the complex conjugate of $H$. For any $x \in$ $H$, the corresponding element of $\bar{H}$ will be denoted by $\bar{x}$. Then $H \ni x \rightarrow \bar{x} \in \bar{H}$ is an antiunitary map. In particular $(\bar{x} \mid \bar{y})=(y \mid x)$ for any $x, y \in H$. For any closed operator $m$ acting on $H, m^{\top}$ will denote the transpose of $m$. By definition $D\left(m^{\top}\right)=\overline{D\left(m^{*}\right)}$ and

$$
m^{\top} \bar{x}=\overline{m^{*} x}
$$

for any $x \in D\left(m^{*}\right)$. For any $m \in B(H), m^{\top}$ is a bounded operator acting on $\bar{H}$ such that $\left(\bar{x} \mid m^{\top} \bar{y}\right)=(y \mid m x)$ for all $x, y \in H$. Clearly $B(H) \ni m \rightarrow m^{\top} \in B(\bar{H})$ is an antiisomorphism of $C^{*}$-algebras. Setting $\overline{\bar{x}}=x$ we identify $\overline{\bar{H}}$ with $H$. With this identification $m^{\top \top}=m$ for any $m \in B(H)$.

Let $W$ be a bounded operator acting on $H \otimes H: W \in B(H \otimes H)$. We shall use the leg numbering notation: $W_{12}=W \otimes I, W_{13}=(I \otimes \Sigma)(W \otimes I)(I \otimes \Sigma)$ and $W_{23}=I \otimes W$. In these formulae $I$ is the unit operator acting on $H$ and $\Sigma \in B(H \otimes H)$ is the flip: $\Sigma(x \otimes y)=y \otimes x$ for any $x, y \in H$. Clearly $W_{12}, W_{13}, W_{23} \in B(H \otimes H \otimes H)$. Similarly for any $V \in B(K \otimes H)$ (where $K$ is another Hilbert space) one introduces $V_{12}, V_{13} \in B(K \otimes H \otimes H)$.

Definition 1.1 Let $H$ be a Hilbert space and $W$ be a unitary operator acting on $H \otimes H$. We say that $W$ is a multiplicative unitary if it satisfies the pentagonal equation

$$
W_{23} W_{12}=W_{12} W_{13} W_{23} \text {. }
$$

Definition 1.2 Let $H$ be a Hilbert space and $W \in B(H \otimes H)$ be a multiplicative unitary. $W e$ say that $W$ is manageable if there exist a positive selfadjoint operator $Q$ acting on $H$ and a unitary operator $\widetilde{W}$ acting on $\bar{H} \otimes H$ such that $\operatorname{ker}(Q)=\{0\}$,

$$
W^{*}(Q \otimes Q) W=Q \otimes Q
$$

and

$$
(x \otimes u|W| z \otimes y)=\left(\bar{z} \otimes Q u|\widetilde{W}| \bar{x} \otimes Q^{-1} y\right)
$$

for any $x, z \in H, y \in D\left(Q^{-1}\right)$ and $u \in D(Q)$. 
Definition 1.3 Let $H$ and $K$ be Hilbert spaces, $W \in B(H \otimes H)$ be a multiplicative unitary and $V$ be a unitary operator acting on $K \otimes H$. We say that $V$ is adapted to $W$ if

$$
W_{23} V_{12}=V_{12} V_{13} W_{23}
$$

Definitions 1.1 and 1.3 are due to Baaj and Skandalis [3]. In their terminology adapted unitaries are called representations. Definition 1.2 contains the condition that in our approach replaces the condition of regularity of [3]. The both conditions have more or less the same implications. However there are strong indications [4] that the condition formulated in Definition 1.2 is satisfied for all multiplicative unitaries related to quantum groups. This is not the case for regularity condition $[1,2]$.

It is well known [3] that for any multiplicative unitary $W$, the dual $\widehat{W}=\Sigma W^{*} \Sigma$ is a multiplicative unitary. We shall prove that the dual of a manageable multiplicative unitary is manageable. The operator $Q$ remains the same.

Proposition 1.4 Let $H$ be a separable Hilbert space, $W \in B(H \otimes H)$ be a manageable multiplicative unitary and $Q$ and $\widetilde{W}$ be the operators related to $W$ in the way described in Definition 1.2. Then

1. $\widetilde{W}$ and $Q^{\top} \otimes Q^{-1}$ commute:

$$
\widetilde{W}\left(Q^{\top} \otimes Q^{-1}\right) \widetilde{W}^{*}=Q^{\top} \otimes Q^{-1}
$$

2. For any $x \in D\left(Q^{-1}\right), z \in D(Q)$ and $y, u \in H$ :

$$
(x \otimes u|W| z \otimes y)=\left(\overline{Q z} \otimes u|\widetilde{W}| \overline{Q^{-1} x} \otimes y\right) .
$$

3. The multiplicative unitary $\widehat{W}=\Sigma W^{*} \Sigma$ is manageable.

\section{Proof:}

Ad 1. Due to (1.3), the left hand side of (1.4) remains unchanged when one replaces $x, y, z$ and $u$ by $Q^{i t} x, Q^{i t} y, Q^{i t} z$ and $Q^{i t} u$ respectively $(t \in \mathbf{R})$. Taking into account (1.1) we get

$$
\begin{gathered}
\left(\bar{z} \otimes Q u|\widetilde{W}| \bar{x} \otimes Q^{-1} y\right)=\left(\left[Q^{\top}\right]^{-i t} \bar{z} \otimes Q^{i t} Q u|\widetilde{W}|\left[Q^{\top}\right]^{-i t} \bar{x} \otimes Q^{i t} Q^{-1} y\right) \\
\widetilde{W}=\left(\left[Q^{\top}\right]^{i t} \otimes Q^{-i t}\right) \widetilde{W}\left(\left[Q^{\top}\right]^{-i t} \otimes Q^{i t}\right)
\end{gathered}
$$

and (1.6) follows.

Ad 2. Assume for the moment, that in addition $y \in D\left(Q^{-1}\right)$ and $u \in D(Q)$. Then our formula follows immediately from Statement 1 and formula (1.4). To get (1.7) in full generality it is sufficient to use the continuity argument.

Ad 3. One can easily verify that $\widehat{W}$ commutes with $Q \otimes Q$. Moreover introducing the unitary $\widetilde{\widehat{W}}=\left(\Sigma \widetilde{W}^{*} \Sigma\right)^{\top \otimes \top}$ we have:

$$
(x \otimes u|\widehat{W}| z \otimes y)=\left(\bar{z} \otimes Q u|\widetilde{\widetilde{W}}| \bar{x} \otimes Q^{-1} y\right)
$$


for any $x, z \in H, y \in D\left(Q^{-1}\right)$ and $u \in D(Q)$. Indeed, using in the forth step formula (1.7) we obtain:

$$
\begin{gathered}
\left(\bar{z} \otimes Q u\left|\left(\Sigma \widetilde{W}^{*} \Sigma\right)^{\top \otimes \top}\right| \bar{x} \otimes Q^{-1} y\right)=\left(x \otimes \overline{Q^{-1} y}\left|\Sigma \widetilde{W}^{*} \Sigma\right| z \otimes \overline{Q u}\right) \\
=\left(\overline{Q^{-1} y} \otimes x\left|\widetilde{W}^{*}\right| \overline{Q u} \otimes z\right)=\overline{\left(\overline{Q u} \otimes z|\widetilde{W}| \overline{Q^{-1} y} \otimes x\right)}=\overline{(y \otimes z|W| u \otimes x)} \\
=\left(u \otimes x\left|W^{*}\right| y \otimes z\right)=\left(x \otimes u\left|\Sigma W^{*} \Sigma\right| z \otimes y\right)
\end{gathered}
$$

and (1.8) follows. It shows, that $\widehat{W}$ is manageable.

Q.E.D.

The main results of the paper are containing in the following theorems:

Theorem 1.5 Let $H$ be a separable Hilbert space, $W \in B(H \otimes H)$ be a manageable multiplicative unitary and

$$
\begin{gathered}
A=\left\{(\omega \otimes \mathrm{id}) W: \omega \in B(H)_{*}\right\}^{\text {norm closure }}, \\
\hat{A}=\left\{(\mathrm{id} \otimes \omega)\left(W^{*}\right): \omega \in B(H)_{*}\right\}^{\text {norm closure }} .
\end{gathered}
$$

Then

1. $A$ and $\hat{A}$ are separable $C^{*}$-algebras acting on $H$ in a non-degenerate way.

2. $W \in M(\hat{A} \otimes A)$.

3. There exists unique $\Delta \in \operatorname{Mor}(A, A \otimes A)$ such that

$$
(\mathrm{id} \otimes \Delta) W=W_{12} W_{13}
$$

$\Delta$ is coassociative: $(\Delta \otimes \mathrm{id}) \Delta=(\mathrm{id} \otimes \Delta) \Delta$. Moreover $\{\Delta(a)(I \otimes b): a, b \in A\}$ and $\{(a \otimes I) \Delta(b): a, b \in A\}$ are linearly dense subsets of $A \otimes A$.

4. There exists a unique closed linear operator $\kappa$ acting on the Banach space $A$ such that $\left\{(\omega \otimes \mathrm{id}) W: \omega \in B(H)_{*}\right\}$ is a core (essential domain) of $\kappa$ and

$$
\kappa((\omega \otimes \mathrm{id}) W)=(\omega \otimes \mathrm{id})\left(W^{*}\right)
$$

for any $\omega \in B(H)_{*}$. The domain $D(\kappa)$ is a subalgebra of $A$ and $\kappa$ is antimultiplicative: for any $a, b \in D(\kappa), \kappa(a b)=\kappa(b) \kappa(a)$. The image $\kappa(D(\kappa))$ coincides with $D(\kappa)^{*}$ and $\kappa\left(\kappa(a)^{*}\right)^{*}=a$ for any $a \in D(\kappa)$.

The operator $\kappa$ admits the following polar decomposition:

$$
\kappa=R \circ \tau_{i / 2}
$$

where $\tau_{i / 2}$ is the analytic generator of a one parameter group $\left(\tau_{t}\right)_{t \in \mathbf{R}}$ of ${ }^{*}$-automorphisms of the $C^{*}$-algebra $A$ and $R$ is an involutive normal ${ }^{1}$ antiautomorphism of $A$ commuting

\footnotetext{
${ }^{1}$ i.e. continuous with respect to the ultra-weak operator topology on $A$, we need not to say that $R$ is norm continuous
} 
with automorphisms $\tau_{t}$ for all $t \in \mathbf{R}$. In particular $D(\kappa)=D\left(\tau_{i / 2}\right)$. R and $\left(\tau_{t}\right)_{t \in \mathbf{R}}$ are uniquely determined.

5. Let $\widetilde{W}$ and $Q$ be operators entering Definition 1.2. Then for any $t \in \mathbf{R}$ and $a \in A$ we have:

$$
\tau_{t}(a)=Q^{2 i t} a Q^{-2 i t}
$$

Moreover using the exponent notation for $R$ (i.e. writing $a^{R}$ instead of $R(a)$ ) we have:

$$
W^{\top \otimes R}=\widetilde{W}^{*} .
$$

It is understood that the group $\left(\tau_{t}\right)_{t \in \mathbf{R}}$ is pointwise continuous: for any $a \in A$, $\left\|\tau_{t}(a)-a\right\| \rightarrow 0$ when $t \rightarrow 0$. Let us recall [9] that the analytical generator $\tau_{i / 2}$ of a (pointwise continuous) one parameter group $\left(\tau_{t}\right)_{t \in \mathbf{R}}$ of *-automorphisms of a $C^{*}$-algebra $A$ is the linear operator acting on $A$ in the following way:

For any $a, b \in A: a \in D\left(\tau_{i / 2}\right)$ and $b=\tau_{i / 2}(a)$ if and only if there exists a mapping $z \mapsto a_{z} \in A$ continuous on the strip $\{z \in \mathbf{C}: \Im z \in[0,1 / 2]\}$ and holomorphic in the interior of this strip such that $a_{t}=\tau_{t}(a)$ for all $t \in \mathbf{R}$ and $a_{i / 2}=b$.

It is known that $\tau_{i / 2}$ is a closed linear mapping, $D\left(\tau_{i / 2}\right)$ is a dense subalgebra and $\tau_{i / 2}$ is multiplicative. Moreover $\tau_{i / 2}(a)^{*} \in D\left(\tau_{i / 2}\right)$ and $\tau_{i / 2}\left(\tau_{i / 2}(a)^{*}\right)^{*}=a$ for any $a \in D\left(\tau_{i / 2}\right)$.

Due to the polar decomposition (1.13) $\kappa$ admits a natural extension to an operator $\tilde{\kappa}$ acting on $M(A) . \tilde{\kappa}$ is the closure of $\kappa$ with respect to the strict topology of $M(A)$. This extension is used at the point 4 of the following theorem.

Theorem 1.6 Let $H$ and $K$ be separable Hilbert spaces, $W \in B(H \otimes H)$ be a manageable multiplicative unitary, $V \in B(K \otimes H)$ be a unitary adapted to $W$ and

$$
B=\left\{(\mathrm{id} \otimes \omega)\left(V^{*}\right): \omega \in B(H)_{*}\right\}^{\text {norm closure }} .
$$

Then, using the notation introduced in the previous theorem we have:

0. $V$ is 'manageable': There exists a unitary $\tilde{V} \in B(\bar{K} \otimes H)$ such that

$$
(r \otimes u|V| s \otimes y)=\left(\bar{s} \otimes Q u|\widetilde{V}| \bar{r} \otimes Q^{-1} y\right)
$$

for any $r, s \in K, y \in D\left(Q^{-1}\right)$ and $u \in D(Q)$.

1. $B$ is a separable $C^{*}$-algebra acting on $K$ in a non-degenerate way.

2. $V \in M(B \otimes A)$.

3. $(\mathrm{id} \otimes \Delta) V=V_{12} V_{13}$.

4. For any $\varphi \in B(K)_{*}$ we have: $(\varphi \otimes \mathrm{id}) V \in D(\tilde{\kappa})$ and

$$
\tilde{\kappa}((\varphi \otimes \mathrm{id}) V)=(\varphi \otimes \mathrm{id})\left(V^{*}\right) .
$$

5. $V^{\top \otimes R}=\widetilde{V}^{*}$. 
6. The $C^{*}$-algebra $B$ is generated by $V \in M(B \otimes A)$ in the sense described in [8]: for any Hilbert space $L$, any $\pi \in \operatorname{Rep}(B, L)$ and any non-degenerate separable $C^{*}$-algebra $D \subset B(L)$ we have:

$$
((\pi \otimes \mathrm{id}) V \in M(D \otimes A)) \Longrightarrow(\pi \in \operatorname{Mor}(B, D))
$$

It turns out that the condition 3 of the above theorem characterizes the adapted operators completely. We have:

Theorem 1.7 Let $H$ and $K$ be separable Hilbert spaces, $W \in B(H \otimes H)$ be a manageable multiplicative unitary and $V$ be a unitary element of $M(C B(K) \otimes A)$. Assume that (id $\otimes \Delta) V=V_{12} V_{13}$. Then $V$ is a unitary adapted to $W$.

\section{The manageability of adapted unitaries}

Throughout this Section $H$ and $K$ are separable Hilbert spaces, $W \in B(H \otimes H)$ is a manageable multiplicative unitary and $V \in M(C B(K) \otimes A)$ is a unitary adapted to $W$. Moreover $Q$ and $\widetilde{W}$ will be the operators related to $W$ in the way described in Definition 1.2 .

We shall prove Statement 0 of Theorem 1.6.

Let $\widetilde{\widetilde{V}}=\left(V^{\top \otimes \top} \otimes I\right)(I \otimes \widetilde{W})\left(V^{* \top \otimes \top} \otimes I\right)\left(I \otimes \widetilde{W}^{*}\right)$. Clearly $\widetilde{\widetilde{V}}$ is a unitary operator acting on $\bar{K} \otimes \bar{H} \otimes H$. We shall prove that $\widetilde{\widetilde{V}}$ is of the form $\widetilde{\widetilde{V}}=\widetilde{V}_{13}$, where $\widetilde{V}$ is a unitary operator acting on $\bar{K} \otimes H$. Next we shall show that the later operator satisfies (1.16).

In the following computations $r, s \in K, x, y \in D\left(Q^{-1}\right)$ and $z, u \in D(Q)$. Let $\nu$ and $\mu$ be normal linear functionals on $B(H)$ such that

$$
\mu(a)=(u|a| y), \quad \nu(a)=\left(Q u|a| Q^{-1} y\right)
$$

for any $a \in B(H)$. According to $(1.4),(x|(\mathrm{id} \otimes \mu) W| z)=(\bar{z}|(\mathrm{id} \otimes \nu) \widetilde{W}| \bar{x})$. It shows that

$$
[(\mathrm{id} \otimes \mu) W]^{\top}=(\mathrm{id} \otimes \nu) \widetilde{W}
$$

By the definition of $\widetilde{\widetilde{V}}$ :

$$
\widetilde{\widetilde{V}}(I \otimes \widetilde{W})=\left(V^{\top \otimes \top} \otimes I\right)(I \otimes \widetilde{W})\left(V^{* \top \otimes \top} \otimes I\right) .
$$

Therefore

$$
\begin{aligned}
& (\mathrm{id} \otimes \mathrm{id} \otimes \nu)[\widetilde{\widetilde{V}}(I \otimes \widetilde{W})]=V^{\top \otimes \top}[I \otimes(\mathrm{id} \otimes \nu) \widetilde{W}] V^{* \top \otimes \top} \\
& =V^{\top \otimes \top}[I \otimes(\mathrm{id} \otimes \mu) W]^{\top \otimes \top} V^{* \top \otimes \top}=\left\{V^{*}[I \otimes(\mathrm{id} \otimes \mu) W] V\right\}^{\top \otimes \top} \\
& =\left\{(\mathrm{id} \otimes \mathrm{id} \otimes \mu) V_{12}^{*} W_{23} V_{12}\right\}^{\top \otimes \top}=\left\{(\mathrm{id} \otimes \mathrm{id} \otimes \mu) V_{13} W_{23}\right\}^{\top \otimes \top},
\end{aligned}
$$


where in the last step we used (1.2). Now we have:

$$
\begin{aligned}
& \left(\bar{s} \otimes \bar{z} \otimes Q u|\widetilde{\widetilde{V}}(I \otimes \widetilde{W})| \bar{r} \otimes \bar{x} \otimes Q^{-1} y\right)=(\bar{s} \otimes \bar{z}|(\mathrm{id} \otimes \mathrm{id} \otimes \nu)[\widetilde{\widetilde{V}}(I \otimes \widetilde{W})]| \bar{r} \otimes \bar{x}) \\
& =\left(\bar{s} \otimes \bar{z}\left|\left\{(\mathrm{id} \otimes \mathrm{id} \otimes \mu) V_{13} W_{23}\right\}^{\top \otimes \top}\right| \bar{r} \otimes \bar{x}\right)=\left(r \otimes x\left|(\mathrm{id} \otimes \mathrm{id} \otimes \mu)\left(V_{13} W_{23}\right)\right| s \otimes z\right) \\
& =\left(r \otimes x \otimes u\left|V_{13} W_{23}\right| s \otimes z \otimes y\right)
\end{aligned}
$$

Let $\varphi$ and $\varphi^{\top}$ be normal linear functionals on $B(K)$ and $B(\bar{K})$ such that

$$
\varphi(m)=(r|m| s), \quad \varphi^{\top}(n)=(\bar{s}|n| \bar{r})
$$

for any $m \in B(K)$ and $n \in B(\bar{K})$. With this notation, the result of the above computations can be written in the following way:

$$
\begin{aligned}
& \left(\bar{z} \otimes Q u\left|\left[\left(\varphi^{\top} \otimes \mathrm{id} \otimes \mathrm{id}\right) \widetilde{\widetilde{V}}\right] \widetilde{W}\right| \bar{x} \otimes Q^{-1} y\right)=(x \otimes u|[I \otimes(\varphi \otimes \mathrm{id}) V] W| z \otimes y) \\
& =\left(x \otimes[(\varphi \otimes \mathrm{id}) V]^{*} u|W| z \otimes y\right)=\left(\overline{Q z} \otimes[(\varphi \otimes \mathrm{id}) V]^{*} u|\widetilde{W}| \overline{Q^{-1} x} \otimes y\right),
\end{aligned}
$$

where in the last step we used (1.7). Finally we have:

$$
\begin{aligned}
\left(\widetilde{W}^{*}\left[\left(\varphi^{\top} \otimes \mathrm{id} \otimes \mathrm{id}\right) \tilde{\widetilde{V}}\right]^{*}\right. & \left.(\bar{z} \otimes Q u) \mid \bar{x} \otimes Q^{-1} y\right) \\
& =\left(\widetilde{W}^{*}\left(\overline{Q z} \otimes[(\varphi \otimes \mathrm{id}) V]^{*} u\right)\left|\left(Q^{\top} \otimes Q^{-1}\right)^{-1}\right| \bar{x} \otimes Q^{-1} y\right) .
\end{aligned}
$$

We know that $\left(Q^{\top} \otimes Q^{-1}\right)^{-1}$ is selfadjoint. The above relation shows that:

$$
\widetilde{W}^{*}\left(\overline{Q z} \otimes[(\varphi \otimes \mathrm{id}) V]^{*} u\right) \in D\left(\left(Q^{\top} \otimes Q^{-1}\right)^{-1}\right)
$$

and

$$
\left(Q^{\top} \otimes Q^{-1}\right)^{-1} \widetilde{W}^{*}\left(\overline{Q z} \otimes[(\varphi \otimes \mathrm{id}) V]^{*} u\right)=\widetilde{W}^{*}\left[\left(\varphi^{\top} \otimes \mathrm{id} \otimes \mathrm{id}\right) \widetilde{\widetilde{V}}^{*}(\bar{z} \otimes Q u) .\right.
$$

Taking into account the commutativity (1.6) we see that

$$
[(\varphi \otimes \mathrm{id}) V]^{*} u \in D(Q)
$$

and

$$
\bar{z} \otimes Q[(\varphi \otimes \mathrm{id}) V]^{*} u=\left[\left(\varphi^{\top} \otimes \mathrm{id} \otimes \mathrm{id}\right) \widetilde{\widetilde{V}}\right]^{*}(\bar{z} \otimes Q u)
$$

The appearance of the factor $\bar{z}$ on the left hand side shows that $\widetilde{\widetilde{V}}$ is of the form $\widetilde{\widetilde{V}}=\widetilde{V}_{13}$ Introducing this information into the last equation we get

$$
Q[(\varphi \otimes \mathrm{id}) V]^{*} u=\left[\left(\varphi^{\top} \otimes \mathrm{id}\right) \widetilde{V}\right]^{*} Q u .
$$

Now we have:

$$
\begin{gathered}
\left(Q[(\varphi \otimes \mathrm{id}) V]^{*} u \mid Q^{-1} y\right)=\left(\left[\left(\varphi^{\top} \otimes \mathrm{id}\right) \widetilde{V}\right]^{*} Q u \mid Q^{-1} y\right) \\
(u|(\varphi \otimes \mathrm{id}) V| y)=\left(Q u\left|\left(\varphi^{\top} \otimes \mathrm{id}\right) \widetilde{V}\right| Q^{-1} y\right)
\end{gathered}
$$

and using (2.1) we obtain (1.16). This way Statement 0 of Theorem 1.6 is proved. 


\section{The algebras generated by adapted unitaries}

The title of this Section refers to the norm closed subspace $B$ of $B(K)$ introduced by (1.15). The aim is to prove that $B$ is a $\mathrm{C}^{*}$-algebra.

We keep the notation used in the previous Section: $H$ and $K$ are separable Hilbert spaces, $W \in B(H \otimes H)$ is a manageable multiplicative unitary, $V \in M(C B(K) \otimes A)$ is a unitary adapted to $W$ and $Q$ and $\widetilde{W}$ are the operators related to $W$ in the way described in Definition 1.2.

For any bounded operator $\rho \in B(H)$ we set:

$$
\|\rho\|_{\mathrm{HS}}=\left[\operatorname{Tr} \rho^{*} Q^{-2} \rho\right]^{\frac{1}{2}}
$$

If $\|\rho\|_{\mathrm{HS}}<\infty$, then $\rho H \subset D\left(Q^{-1}\right)$ and $Q^{-1} \rho$ is an operator of Hilbert-Schmidt class. Moreover choosing an orthonormal basis $\left(e_{n}\right)_{n=1,2, \ldots}$ in $H$ we have:

$$
\|\rho\|_{\mathrm{HS}}^{2}=\sum_{n=1}^{\infty}\left\|Q^{-1} \rho e_{n}\right\|^{2} .
$$

For any $x \in H$ we consider the operator

$$
\theta_{x}: \mathbf{C} \ni \lambda \longmapsto \lambda x \in H
$$

Clearly $\theta_{x} \in B(\mathbf{C}, H)$. The hermitian adjoint of $\theta_{x}$ acts in the following way:

$$
\theta_{x}^{*}: H \ni z \longmapsto(x \mid z) \in \mathbf{C} .
$$

The reader should notice that the Dirac dyad $\mid x)\left(y \mid=\theta_{x} \theta_{y}{ }^{*}\right.$. We shall also use the operators $\theta$ in the tensor product context. For example: $\left(I \otimes \theta_{x}^{*}\right)(z \otimes y)=z(x \mid y)$, $\left(\theta_{x} \otimes I\right) y=x \otimes y$ and so on.

The following proposition plays an important role in this paper:

Proposition 3.1 Let $u \in D(Q)$ and $\rho \in B(H)$ Then

$$
\left\|\left(I \otimes \theta_{u}^{*}\right) V(I \otimes \rho)\right\| \leq\|Q u\| \cdot\|\rho\|_{\mathrm{HS}} .
$$

Proof: Let $\left(e_{n}\right)_{n=1,2, \ldots}$ be an orthonormal basis in $H$. Then any vector $\psi \in K \otimes H$ is of the form

$$
\psi=\sum_{n=1}^{\infty} \psi_{n} \otimes e_{n},
$$

where $\psi_{n}$ are elements of $K$ such that

$$
\sum_{n=1}^{\infty}\left\|\psi_{n}\right\|^{2}=\|\psi\|^{2}<\infty .
$$

We may assume that $\|\rho\|_{\text {HS }}<\infty$. Then $\rho H \subset D\left(Q^{-1}\right)$ and for any $y \in K$ we have:

$$
\left(y\left|\left(I \otimes \theta_{u}^{*}\right) V(I \otimes \rho)\right| \psi\right)=(y \otimes u|V(I \otimes \rho)| \psi)
$$




$$
=\sum_{n=1}^{\infty}\left(y \otimes u|V| \psi_{n} \otimes \rho e_{n}\right)=\sum_{n=1}^{\infty}\left(\overline{\psi_{n}} \otimes Q u|\widetilde{V}| \bar{y} \otimes Q^{-1} \rho e_{n}\right) .
$$

Therefore

$$
\begin{aligned}
\left|\left(y\left|\left(I \otimes \theta_{u}^{*}\right) V(I \otimes \rho)\right| \psi\right)\right| & \leq \sum_{n=1}^{\infty}\left\|\psi_{n}\right\| \cdot\|Q u\| \cdot\|y\| \cdot\left\|Q^{-1} \rho e_{n}\right\| \\
& \leq\|y\| \cdot\|Q u\| \cdot\left[\sum_{n=1}^{\infty}\left\|\psi_{n}\right\|^{2}\right]^{\frac{1}{2}}\left[\sum_{n=1}^{\infty}\left\|Q^{-1} \rho e_{n}\right\|^{2}\right]^{\frac{1}{2}} \\
& =\|y\| \cdot\|Q u\| \cdot\|\psi\| \cdot\|\rho\|_{\mathrm{HS}}
\end{aligned}
$$

and (3.1) follows.

Q.E.D.

It will be useful to have a criterion selecting $\rho \in B(H)$ with finite HS-norm.

Proposition 3.2 Let $\rho \in B(H)$. Then $\|\rho\|_{\mathrm{HS}}<\infty$ if and only if there exists a vector $\Psi(\rho) \in \bar{H} \otimes H$ such that

$$
(u \mid \rho z)=(\bar{z} \otimes Q u \mid \Psi(\rho))
$$

for any $u \in D(Q)$ and $z \in H . \Psi(\rho)$ is uniquely determined by $\rho$. The correspondence $\rho \mapsto \Psi(\rho)$ is linear and isometric:

$$
\|\Psi(\rho)\|=\|\rho\|_{\mathrm{HS}}
$$

for any $\rho \in B(H)$ with finite HS-norm.

Proof: The statement follows immediately from the well known $1-1$ correspondence between $\bar{H} \otimes H$ and the Hilbert space of all Hilbert-Schmidt class operators acting on $H$.

Q.E.D.

Example 1: Let $x \in H$ and $y \in D\left(Q^{-1}\right)$. Then $\left.\rho=\mid y\right)\left(x \mid=\theta_{y} \theta_{x}^{*}\right.$ is of finite HS-norm. Indeed for any $u \in D(Q)$ and $z \in H$ we have:

$$
(u \mid \rho z)=(u \mid y)(x \mid z)=\left(\bar{z} \otimes Q u \mid \bar{x} \otimes Q^{-1} y\right)
$$

and $\Psi(\rho)=\bar{x} \otimes Q^{-1} y \in \bar{H} \otimes H$.

Example 2: Let $x \in H$ and $y \in D\left(Q^{-1}\right)$. Then $\rho=\left(\theta_{x}^{*} \otimes I\right) W\left(I \otimes \theta_{y}\right)$ is of finite HS-norm. Indeed for any $u \in D(Q)$ and $z \in H$ we have:

$$
(u \mid \rho z)=(x \otimes u|W| z \otimes y)=\left(\bar{z} \otimes Q u|\widetilde{W}| \bar{x} \otimes Q^{-1} y\right)
$$

and $\Psi(\rho)=\widetilde{W}\left(\bar{x} \otimes Q^{-1} y\right) \in \bar{H} \otimes H$.

In the next example we shall use the antiunitary operator $J$ acting on $\bar{H} \otimes H$ according to the formula:

$$
J(\bar{x} \otimes y)=\bar{y} \otimes x
$$

for any $x, y \in H$. 
Example 3: Let $x \in H$ and $y \in D\left(Q^{-1}\right)$. Then $\rho=\left(I \otimes \theta_{x}^{*}\right) W^{*}\left(\theta_{y} \otimes I\right)$ is of finite HS-norm. Indeed for any $u \in D(Q)$ and $z \in H$ we have $(\operatorname{cf}(1.7))$ :

$$
\begin{aligned}
(u \mid \rho z) & =\left(u \otimes x\left|W^{*}\right| y \otimes z\right)=\overline{(y \otimes z|W| u \otimes x)} \\
& =\overline{\left(\overline{Q u} \otimes z|\widetilde{W}| \overline{Q^{-1} y} \otimes x\right)}=\left(\widetilde{W}\left(\overline{Q^{-1} y} \otimes x\right) \mid \overline{Q u} \otimes z\right) \\
& =\left(\widetilde{W} J\left(\bar{x} \otimes Q^{-1} y\right) \mid J(\bar{z} \otimes Q u)\right)=\left(\bar{z} \otimes Q u|J \widetilde{W} J| \bar{x} \otimes Q^{-1} y\right)
\end{aligned}
$$

and $\Psi(\rho)=J \widetilde{W} J\left(\bar{x} \otimes Q^{-1} y\right) \in \bar{H} \otimes H$.

In what follows, $\mathcal{E}_{1}\left(\mathcal{E}_{2}\right.$ and $\mathcal{E}_{3}$ respectively) will denote the set of all $\rho \in B(H)$ of the form considered in Example 1 (Example 2 and Example 3 respectively). It is very important that each of the three sets is linearly HS-dense in $\left\{\rho \in B(H):\|\rho\|_{\mathrm{HS}}<\infty\right\}$ : For any $\rho \in B(H)$ with finite HS-norm, there exists a sequence $\rho_{n}$ of linear combinations of elements of $\mathcal{E}_{k}$ such that $\left\|\rho-\rho_{n}\right\|_{\mathrm{HS}} \rightarrow 0$. Indeed

$$
\begin{aligned}
& \left\{\Psi(\rho): \begin{array}{l}
\rho \text { is a linear combina- } \\
\text { tion of elements of } \mathcal{E}_{1}
\end{array}\right\}=\bar{H} \otimes_{\text {alg }} D(Q) \\
& \left\{\Psi(\rho): \begin{array}{l}
\rho \text { is a linear combina- } \\
\text { tion of elements of } \mathcal{E}_{2}
\end{array}\right\}=\widetilde{W}\left(\bar{H} \otimes_{\text {alg }} D(Q)\right) \\
& \left\{\Psi(\rho): \begin{array}{l}
\rho \text { is a linear combina- } \\
\text { tion of elements of } \mathcal{E}_{3}
\end{array}\right\}=J \widetilde{W} J\left(\bar{H} \otimes_{\text {alg }} D(Q)\right)
\end{aligned}
$$

and all three sets on the right hand side are dense in $\bar{H} \otimes H$.

Proposition 3.3 Let $\rho \in B(H)$ be an operator with finite HS-norm, $u \in D(Q)$ and $z \in H$. Then

$$
\left(I \otimes \theta_{u}^{*}\right) V(I \otimes \rho) V^{*}\left(I \otimes \theta_{z}\right)=\left(\mathrm{id} \otimes \omega_{\rho}\right) V^{*},
$$

where $\omega_{\rho}$ is a normal functional defined on $B(H)$ such that

$$
\omega_{\rho}(a)=\left(\bar{z} \otimes Q u\left|\widetilde{W}\left(a^{\top} \otimes I\right) \widetilde{W}^{*}\right| \Psi(\rho)\right)
$$

for any $a \in B(H)$.

Proof: Let $L H S(\rho)(R H S(\rho)$ respectively) denote the left (right respectively) hand side of (3.3). By virtue of (3.1)

$$
\|L H S(\rho)\| \leq\|Q u\|\|z\|\|\rho\|_{\mathrm{HS}} .
$$

On the other hand it is obvious that the norm of the functional $\omega_{\rho}$ is majorized by $\|Q u\|\|z\|\|\Psi(\rho)\|$, so we have:

$$
\|R H S(\rho)\| \leq\|Q u\|\|z\|\|\rho\|_{\mathrm{HS}} .
$$

Due to these estimates the set of $\rho$ satisfying (3.3) is HS-closed. We know that $\mathcal{E}_{2}$ is linearly HS-dense in the set of all operators with finite HS-norm. Therefore it is sufficient 
to prove (3.3) for $\rho$ of the form considered in Example 2. Let $\rho=\left(\theta_{x}^{*} \otimes I\right) W\left(I \otimes \theta_{y}\right)$, where $x \in H$ and $y \in D\left(Q^{-1}\right)$. Then $\Psi(\rho)=\widetilde{W}\left(\bar{x} \otimes Q^{-1} y\right)$ and

$$
\begin{aligned}
\omega_{\rho}(a) & =\left(\bar{z} \otimes Q u\left|\widetilde{W}\left(a^{\top} \otimes I\right)\right| \bar{x} \otimes Q^{-1} y\right)=\left(\bar{z} \otimes Q u|\widetilde{W}| \overline{a^{*} x} \otimes Q^{-1} y\right) \\
& =\left(a^{*} x \otimes u|W| z \otimes y\right)=(x \otimes u|(a \otimes I) W| z \otimes y) .
\end{aligned}
$$

Therefore for any $r, s \in K$ we have:

$$
\left(r\left|\left(\mathrm{id} \otimes \omega_{\rho}\right) V^{*}\right| s\right)=\left(r \otimes x \otimes u\left|V_{12}^{*} W_{23}\right| s \otimes z \otimes y\right) .
$$

According to (1.5), $V_{12}^{*} W_{23}=V_{13} W_{23} V_{12}^{*}$. Inserting this information into the right hand side we get

$$
\begin{aligned}
\left(r\left|\left(\mathrm{id} \otimes \omega_{\rho}\right) V^{*}\right| s\right) & =\left(r \otimes x \otimes u\left|V_{13} W_{23} V_{12}^{*}\right| s \otimes z \otimes y\right) \\
& =\left(r \otimes u\left|V\left(I \otimes \theta_{x}^{*} \otimes I\right) W_{23}\left(I \otimes I \otimes \theta_{y}\right) V^{*}\right| s \otimes z\right) \\
& =\left(r \otimes u\left|V(I \otimes \rho) V^{*}\right| s \otimes z\right)
\end{aligned}
$$

and (3.3) follows.

Q.E.D.

Inserting in (3.3) $\rho$ of the form considered in Example 1 we obtain

Proposition 3.4 Let $x, z \in H, y \in D\left(Q^{-1}\right)$ and $u \in D(Q)$. Consider normal functionals $\mu, \nu, \omega \in B(H)_{*}$ introduced by the formulae

$$
\begin{aligned}
\mu(a) & =(u \mid a y), \\
\nu(a) & =(x \mid a z), \\
\omega(a) & =\left(\bar{z} \otimes Q u\left|\widetilde{W}\left(a^{\top} \otimes I\right) \widetilde{W}^{*}\right| \bar{x} \otimes Q^{-1} y\right),
\end{aligned}
$$

where a runs over $B(H)$. Then we have:

$$
(\mathrm{id} \otimes \mu) V(\mathrm{id} \otimes \nu)\left(V^{*}\right)=(\mathrm{id} \otimes \omega)\left(V^{*}\right) .
$$

Now we are able to prove Statement 1 of Theorem 1.6. Let $B$ be the norm closed subspace of $B(K)$ introduced by (1.15). It is well known that the functionals $\mu$ defined by (3.4), where $u$ and $y$ run over a dense subsets of $H$ form a linearly dense subset of $B(H)_{*}$. The same holds for functionals $\nu$ introduced by (3.5). A moment of reflection shows that also the set of functionals $\omega$ of the form (3.6) is linearly dense in $B(H)_{*}$. Therefore in the formula (3.7), the first factor on the left hand side runs over a linearly dense subset of $B^{*}$, whereas the second factor and the right hand side run over a linearly dense subset of $B$. Formula (3.7) shows that $B^{*} B$ is a linearly dense subset of $B$. Since $B^{*} B$ is ${ }^{*}$-invariant, so is $B: B^{*}=B$. Now we have: $B B=B^{*} B \subset B$. It means that $B$ is a ${ }^{*}$-subalgebra of $B(K)$.

Let $r \in K$ and $r \neq 0$. Then for any non-zero $z \in H, r \otimes z \neq 0, V^{*}(r \otimes z) \neq 0$ and there exist $s \in K$ and $x \in H$ such that $\left(s \otimes x\left|V^{*}\right| r \otimes z\right) \neq 0$. Let $\nu$ be the normal 
functional on $B(H)$ defined by $\nu(a)=(x \mid a z)$ for all $a \in B(H)$ and $b=($ id $\otimes \nu)\left(V^{*}\right)$ Then $b \in B,(s \mid b r)=\left(s \otimes x\left|V^{*}\right| r \otimes z\right) \neq 0$ and $b r \neq 0$. It shows that $B$ acts on $K$ in a non-degenerate way. Since $B(H)_{*}$ is separable, so is $B$. Statement 1 of Theorem 1.6 is proved.

Let $K=H$ and $V=W$. Comparing (1.2) and (1.5) we see that $V=W$ is a unitary adapted to $W$. In this particular case $B$ coincides with $\hat{A}$ introduced by $(1.10)$ and consequently $\hat{A}$ is a separable $\mathrm{C}^{*}$-algebra acting on $H$ in a non-degenerate way. Replacing $W$ by its dual $\widehat{W}=\Sigma W^{*} \Sigma$ we get the same result for $A$ introduced by (1.9). Statement 1 of Theorem 1.5 is proved.

\section{Adapted unitaries as multipliers}

In this Section we shall prove the Statements 2 of Theorems 1.5 and 1.6. We shall use the notation introduced in Section 2. We already know that $B$ introduced by (1.15) is a $\mathrm{C}^{*}$-algebra. Applying the hermitian conjugation to the both sides of (1.15) we obtain an alternative definition of $B$ :

$$
B=\left\{(\text { id } \otimes \omega) V: \omega \in B(H)_{*}\right\}^{\text {norm closure }} .
$$

We recall, that the algebra of all compact operators acting on the Hilbert space $H$ is denoted by $C B(H)$. This is the smallest norm closed subspace of $B(H)$ containing all one dimensional operators $\theta_{z} \theta_{x}^{*}(z, x \in H)$. We start with the following interesting

Proposition 4.1 Let $z \in H, u \in D(Q)$ and $\rho \in B(H),\|\rho\|_{\mathrm{HS}}<\infty$. Then

$$
\left(I \otimes \theta_{z} \theta_{u}^{*}\right) V(I \otimes \rho) \in B \otimes C B(H) .
$$

Moreover the set of all elements of the form (4.2) is linearly dense in $B \otimes C B(H)$.

Proof: By virtue of (3.1) we have

$$
\left\|\left(I \otimes \theta_{z} \theta_{u}^{*}\right) V(I \otimes \rho)\right\| \leq\|z\| \cdot\|Q u\| \cdot\|\rho\|_{\mathrm{HS}} .
$$

Due to this estimates the set of $\rho$ satisfying (4.2) is HS-closed. We know that $\mathcal{E}_{1}$ is linearly HS-dense in the set of all operators with finite HS-norm. Therefore it is sufficient to prove (4.2) for $\rho$ of the form considered in Example 1.

Let $\rho=\theta_{y} \theta_{x}^{*}$, where $x \in H$ and $y \in D\left(Q^{-1}\right)$. Then

$$
\left(I \otimes \theta_{z} \theta_{u}^{*}\right) V(I \otimes \rho)=(\mathrm{id} \otimes \omega) V \otimes \theta_{z} \theta_{x}^{*}
$$

where $\omega$ is the normal functional on $B(H)$ such that $\omega(a)=(u \mid a y)$ for all $a \in B(H)$. By virtue of (4.1), the right hand side of the above equation belongs to $B \otimes_{\text {alg }} C B(H)$ and (4.2) follows. To end the proof one should notice that elements of the form (id $\otimes \omega) V$ $\left(\theta_{z} \theta_{x}^{*}\right.$ respectively) are linearly dense in $B(C B(H)$ respectively).

Q.E.D. 
Proposition 4.2 Let $u, x, y, z \in H$. Then

$$
\left(I \otimes \theta_{z} \theta_{u}^{*} \otimes \theta_{x}^{*}\right) V_{12} W_{23}^{*}\left(I \otimes \theta_{y} \otimes I\right) \in B \otimes C B(H)
$$

Moreover the set of all elements of the form (4.4) is linearly dense in $B \otimes C B(H)$.

Proof: We may additionally assume that $u \in D(Q)$ and $y \in D\left(Q^{-1}\right)(D(Q)$ and $D\left(Q^{-1}\right)$ are dense in $H$. Let $\rho=\left(I \otimes \theta_{x}^{*}\right) W^{*}\left(\theta_{y} \otimes I\right)$. Then $\|\rho\|_{\mathrm{HS}}<\infty$ (cf Example 3$)$. Inserting this particular value of $\rho$ into (4.2) we obtain

$$
\left(I \otimes \theta_{z} \theta_{u}^{*}\right) V(I \otimes \rho)=\left(I \otimes \theta_{z} \theta_{u}^{*} \otimes \theta_{x}^{*}\right) V_{12} W_{23}^{*}\left(I \otimes \theta_{y} \otimes I\right)
$$

and (4.4) follows. Taking into account the estimate (4.3) and the linear HS-density of $\mathcal{E}_{3}$ in the space of all operators with finite HS-norm we see that the closed linear span of all elements of the form (4.4) coincides with that of all elements of the form (4.2) i.e. with $B \otimes C B(H)$. It shows that the set of all elements of the form (4.4) is linearly dense in $B \otimes C B(H)$.

Q.E.D.

According to (1.5) $V_{12} W_{23}^{*}=W_{23}^{*} V_{12} V_{13}$. Therefore

$$
\begin{aligned}
\left(I \otimes \theta_{z} \theta_{u}^{*} \otimes \theta_{x}^{*}\right) V_{12} W_{23}^{*}\left(I \otimes \theta_{y} \otimes I\right) & =\left(I \otimes \theta_{z} \theta_{u}^{*} \otimes \theta_{x}^{*}\right) W_{23}^{*} V_{12} V_{13}\left(I \otimes \theta_{y} \otimes I\right) \\
& =\left(I \otimes \theta_{z} \theta_{u}^{*} \otimes \theta_{x}^{*}\right) W_{23}^{*} V_{12}\left(I \otimes \theta_{y} \otimes I\right) V .
\end{aligned}
$$

Proposition 4.3 Let $u, x, y, z \in H$. Then

$$
\left(I \otimes \theta_{z} \theta_{u}^{*} \otimes \theta_{x}^{*}\right) W_{23}^{*} V_{12}\left(I \otimes \theta_{y} \otimes I\right) \in B \otimes C B(H)
$$

Moreover the set of all elements of the form (4.6) is linearly dense in $B \otimes C B(H)$.

Proof: At first we notice that the element (4.6) may be written in the form

$$
\begin{aligned}
\left(I \otimes \theta_{z} \theta_{u}^{*} \otimes \theta_{x}^{*}\right) W_{23}^{*} V_{12}\left(I \otimes \theta_{y} \otimes I\right) & =\left(I \otimes \theta_{z}\right)\left(I \otimes\left(\theta_{u}^{*} \otimes \theta_{x}^{*}\right) W^{*}\right)(V \otimes I)\left(I \otimes \theta_{y} \otimes I\right) \\
& =\left(I \otimes \theta_{z}\right)\left(I \otimes \theta_{W(u \otimes x)}^{*}\right)(V \otimes I)\left(I \otimes \theta_{y} \otimes I\right) .
\end{aligned}
$$

The set $W\left(H \otimes_{\text {alg }} H\right)$ is dense in $H \otimes H$. So is the set $H \otimes_{\text {alg }} H$. Therefore replacing $W(u \otimes x)$ by $u \otimes x$ we do not change the closed linear span: the closed linear span of all elements of the form (4.6) coincides with that of all elements of the form

$$
\left(I \otimes \theta_{z}\right)\left(I \otimes \theta_{u \otimes x}^{*}\right)(V \otimes I)\left(I \otimes \theta_{y} \otimes I\right)=(\mathrm{id} \otimes \omega) V \otimes \theta_{z} \theta_{x}^{*}
$$

where $\omega$ is the normal functional on $B(H)$ such that $\omega(a)=(u \mid a y)$ for all $a \in B(H)$. We already know (cf the last part of the proof of Proposition 4.1) that these elements belong to $B \otimes C B(H)$ and that their closed linear span coincides with $B \otimes C B(H)$.

Q.E.D.

Combining Propositions 4.2 and 4.3 with the formula (4.5) we see that

$$
B \otimes C B(H)=(B \otimes C B(H)) V .
$$


It means that $V$ is a right multiplier of $B \otimes C B(H)$. Multiplying both sides of the above formula by $V^{*}$ from the left we get $(B \otimes C B(H)) V^{*}=B \otimes C B(H)$. It shows that $V^{*}$ is a right multiplier of $B \otimes C B(H)$. Consequently $V$ is a left multiplier of $B \otimes C B(H)$ and

$$
V \in M(B \otimes C B(H)) .
$$

Using this result in the special case $K=H, V=W$ (cf the end of Section 2) we get

$$
W \in M(\hat{A} \otimes C B(H)) .
$$

Replacing $W$ by its dual $\widehat{W}=\Sigma W^{*} \Sigma$ we obtain $\Sigma W^{*} \Sigma \in M(A \otimes C B(H))$ and

$$
W \in M(C B(H) \otimes A) .
$$

Now we may use the beautiful argument of Baaj and Skandalis (cf [3, page 441]): According to (1.5)

$$
V_{13}=V_{12}^{*} W_{23} V_{12} W_{23}^{*}
$$

By virtue of (4.7) and (4.8), $V_{12}$ and $W_{23}$ belong to $M(B \otimes C B(H) \otimes A)$. Therefore $V_{13} \in M(B \otimes C B(H) \otimes A)$ and $V \in M(B \otimes A)$. Statement 2 of Theorem 1.6 is proved. In the special case $K=H, V=W$ we obtain Statement 2 of Theorem 1.5.

Now we can prove Statement 6 of Theorem 1.6. Let $L$ be a separable Hilbert space, $D \subset B(L)$ be a separable $\mathrm{C}^{*}$-algebra acting on $L$ in a non-degenerate way and $\pi \in \operatorname{Rep}(B, L)$. Assume that

$$
(\pi \otimes \mathrm{id}) V \in M(D \otimes A) .
$$

We have to show that $\pi \in \operatorname{Mor}(B, D)$, i.e. that $\pi(B) D$ is a linearly dense subset of $D$ (cf $\left[8\right.$, formula (1.5)]). According to (4.9) $(\pi \otimes \mathrm{id}) V^{*} \in M(D \otimes A)$, and for any functional $\omega \in B(H)_{*}$,

$$
\pi\left((\mathrm{id} \otimes \omega) V^{*}\right)=(\mathrm{id} \otimes \omega)(\pi \otimes \mathrm{id}) V^{*} \in M(D) .
$$

It shows that $\pi(B) \subset M(D)$ and consequently $\pi(B) D \subset D$. To prove the density we shall use the unitarity of (4.9). Let $d \in D, a \in A$ and $\omega \in B(H)_{*}$. Then

$$
(\mathrm{id} \otimes \omega)\left(\left((\pi \otimes \mathrm{id}) V^{*}\right)(d \otimes a)\right)=\pi\left((\mathrm{id} \otimes \omega)\left[V^{*}(I \otimes a)\right]\right) d \in \pi(B) D .
$$

Due to the unitarity of (4.9) the set

$$
\left\{\left((\pi \otimes \mathrm{id}) V^{*}\right)(d \otimes a): d \in D, a \in A\right\}
$$

is linearly dense in $D \otimes A$. Therefore the set of elements of the form (4.10) is linearly dense in $D$ and the Statement follows. 


\section{Comultiplication}

In this Section we shall prove Statements 3 of Theorems 1.5 and 1.6. Moreover the last line will contain the proof of Theorem 1.7. Let $W$ be a manageable multiplicative unitary and $A$ be the $\mathrm{C}^{*}$-algebra introduced by (1.9). For any $a \in A$ we set:

$$
\Delta(a)=W(a \otimes I) W^{*} .
$$

With this notation we have:

Proposition 5.1 $\{\Delta(a)(I \otimes b): a, b \in A\}$ and $\{(a \otimes I) \Delta(b): a, b \in A\}$ are linearly dense subsets of the algebra $A \otimes A$.

Proof: Let $\omega \in B(H)_{*}$ and $a=(\omega \otimes \mathrm{id}) W$. Then $a \in A$ and using in the last step (1.2) we obtain

$$
\begin{aligned}
\Delta(a) & =\Delta((\omega \otimes \mathrm{id}) W)=W[(\omega \otimes \mathrm{id}) W \otimes I] W^{*} \\
& =(\omega \otimes \mathrm{id} \otimes \mathrm{id})\left(W_{23} W_{12} W_{23}^{*}\right)=(\omega \otimes \mathrm{id} \otimes \mathrm{id})\left(W_{12} W_{13}\right)
\end{aligned}
$$

We shall use products $\omega m$ and $m \omega$, where $\omega \in B(H)_{*}$ and $m \in C B(H)$. By definition $\omega m$ and $m \omega$ are elements of $B(H)_{*}$ such that $(\omega m)(n)=\omega(m n)$ and $(m \omega)(n)=$ $\omega(n m)$ for any $n \in B(H)$. It is well known (see [7, Thm 16.1] that

$$
\begin{aligned}
& \left\{\omega m: \omega \in B(H)_{*}, m \in C B(H)\right\}=B(H)_{*}, \\
& \left\{m \omega: \omega \in B(H)_{*}, m \in C B(H)\right\}=B(H)_{*} .
\end{aligned}
$$

Let

$$
\Gamma=\{\Delta(a)(I \otimes b): a, b \in A\}^{\substack{\text { closed linear } \\ \text { envelope }}}
$$

We compute:

$$
\begin{aligned}
& \Gamma=\left\{\Delta((m \omega \otimes \mathrm{id}) W)(I \otimes b): \omega \in B(H)_{*}, m \in C B(H), b \in A\right\}^{\substack{\text { closed linear } \\
\text { envelope }}} \\
& =\left\{(\omega \otimes \mathrm{id} \otimes \mathrm{id})\left(W_{12} W_{13}(m \otimes I \otimes b)\right): \omega \in B(H)_{*}, m \in C B(H), b \in A\right\}^{\substack{\text { closed linear } \\
\text { envelope }}} \\
& =\left\{(\omega \otimes \mathrm{id} \otimes \mathrm{id})\left(W_{12} W_{13} T_{13}\right): \omega \in B(H)_{*}, T \in C B(H) \otimes A\right\}^{\substack{\text { closed linear } \\
\text { envelope }}}
\end{aligned}
$$

We know (cf (4.8)) that $W$ is a unitary element of $M(C B(H) \otimes A)$. Therefore $W(C B(H) \otimes A)=C B(H) \otimes A$ and

$$
\begin{aligned}
& \Gamma=\left\{(\omega \otimes \mathrm{id} \otimes \mathrm{id})\left(W_{12} T_{13}\right): \omega \in B(H)_{*}, T \in C B(H) \otimes A\right\}^{\substack{\text { closed linear } \\
\text { envelope }}} \\
& =\left\{(\omega \otimes \mathrm{id} \otimes \mathrm{id})\left(W_{12}(m \otimes I \otimes b)\right): \omega \in B(H)_{*}, m \in C B(H), b \in A\right\}^{\substack{\text { closed linear } \\
\text { envelope }}} \\
& =\left\{(m \omega \otimes \mathrm{id}) W \otimes b: \omega \in B(H)_{*}, m \in C B(H), b \in A\right\}^{\substack{\text { closed linear } \\
\text { envelope }}}=A \otimes A
\end{aligned}
$$


In the similar way one can show that

$$
\{(a \otimes I) \Delta(b): a, b \in A\}^{\substack{\text { closed linear } \\ \text { envelope }}}=A \otimes A .
$$

Q.E.D.

Now the proof of Statement 3 of Theorems 1.5 is very simple: Let $a \in A$. Then for any $b, c \in A$ we have: $\Delta(a)(c \otimes b)=\Delta(a)(I \otimes b)(c \otimes I) \in A \otimes A$. It shows that $\Delta(a)$ is a left multiplier of $A \otimes A$. Replacing $a$ by $a^{*}$ we see that $\Delta(a)$ is a right multiplier and $\Delta(a) \in M(A \otimes A)$. Clearly the mapping

$$
A \ni a \longrightarrow \Delta(a) \in M(A \otimes A)
$$

is a *algebra homomorphism. By virtue of Proposition 5.1 this homomorphism is nondegenerate: $\Delta(A)(A \otimes A)$ is linearly dense in $A \otimes A$. It means, that $\Delta \in \operatorname{Mor}(A, A \otimes A)$.

Let $a \in A$. It follows immediately from the definition (5.1), that

$$
\begin{aligned}
& (\Delta \otimes \mathrm{id}) \Delta(a)=W_{12} W_{13}(a \otimes I \otimes I) W_{13}^{*} W_{12}^{*}, \\
& (\mathrm{id} \otimes \Delta) \Delta(a)=W_{23} W_{12}(a \otimes I \otimes I) W_{12}^{*} W_{23}^{*} .
\end{aligned}
$$

Obviously $W_{23}$ commutes with $a \otimes I \otimes I$, so the pentagonal equation (1.2) shows that the right hand sides of the above relations coincide and the coassociativity of $\Delta$ follows.

Formula (1.11) follows immediately from definition (5.1) and pentagonal relation (1.2):

$$
(\mathrm{id} \otimes \Delta) W=W_{23} W_{12} W_{23}^{*}=W_{12} W_{13} .
$$

In the same way, using (1.5) we prove Statement 3 of Theorem 1.6:

$$
(\mathrm{id} \otimes \Delta) V=W_{23} V_{12} W_{23}^{*}=V_{12} V_{13}
$$

To prove Theorem 1.7 it is sufficient to write the above equalities in another order:

$$
W_{23} V_{12} W_{23}^{*}=(\mathrm{id} \otimes \Delta) V=V_{12} V_{13} .
$$

\section{Scaling group}

In this Section we introduce the scaling group $\left(\tau_{t}\right)_{t \in \mathbf{R}}$ appearing in Statement 4 of Theorem 1.5.

Proposition 6.1 With the notation introduced in Section 1, we have:

1. There exists one parameter group $\left(\tau_{t}\right)_{t \in \mathbf{R}}$ of automorphisms of $A$ such that

$$
\tau_{t}(a)=Q^{2 i t} a Q^{-2 i t}
$$

for any $a \in A$ and $t \in \mathbf{R}$. The group is pointwise continuous: $\left\|\tau_{t}(a)-a\right\| \rightarrow 0$ when $t \rightarrow 0$. 
2. Let $\tau_{i / 2}$ be the analytic generator of $\left(\tau_{t}\right)_{t \in \mathbf{R}}$. Then for any $\omega \in B(H)_{*}$ :

$$
(\omega \otimes \mathrm{id}) W \in D\left(\tau_{i / 2}\right)
$$

and

$$
\tau_{i / 2}((\omega \otimes \mathrm{id}) W)=\left(\omega^{\top} \otimes \mathrm{id}\right) \widetilde{W}
$$

where $\omega^{\top} \in B(\bar{H})_{*}$ is related to $\omega$ by the formula: $\omega^{\top}\left(m^{\top}\right)=\omega(m)$ for any $m \in B(H)$.

3. $\mathcal{A}=\left\{(\omega \otimes \mathrm{id}) W: \omega \in B(H)_{*}\right\}$ is a core of $\tau_{i / 2}$.

Remark: In particular Statement 2 shows that $\left(\omega^{\top} \otimes \mathrm{id}\right) \widetilde{W} \in A$ for any $\omega^{\top} \in B(\bar{H})_{*}$. Proof:

Ad. 1. Let $\omega \in B(H)_{*}$ and $t \in \mathbf{R}$. By virtue of (1.3)

$$
Q^{2 i t}((\omega \otimes \mathrm{id}) W) Q^{-2 i t}=\left(\omega_{t} \otimes \mathrm{id}\right) W
$$

where $\omega_{t}$ is a normal functional on $B(H)$ introduced by the formula

$$
\omega_{t}(m)=\omega\left(Q^{-2 i t} m Q^{2 i t}\right)
$$

for any $m \in B(H)$. The reader should notice that $\left\|\omega_{t}-\omega\right\|_{B(H)_{*}} \rightarrow 0$, when $t \rightarrow 0$.

Formula (6.3) shows that for any $a \in A, \tau_{t}(a)$ introduced by (6.1) belongs to $A$ and that $\tau_{t}(a)$ is a continuous (in the sense of the norm topology) function of $t$. Clearly $\left(\tau_{t}\right)_{t \in \mathbf{R}}$ is a one-parameter automorphism group of $A$.

Ad. 2. Analytic generators are closed operators. Therefore it is sufficient to prove the statement for $\omega$ running over a linearly dense subset of $B(H)_{*}$. In particular we may assume that $\omega(m)=(x \mid m z)$, where $x \in D(Q)$ and $z \in D\left(Q^{-1}\right)$ are fixed vectors $(m \in B(H))$.

For any $t \in \mathbf{C}$ such that $\Im t \in[0,1 / 2]$ and any $m \in B(H)$ we set

$$
\omega_{t}(m)=\left(Q^{2 i \bar{t}} x \mid m Q^{2 i t} z\right)
$$

Then the mapping $t \mapsto \omega_{t} \in B(H)_{*}$ is continuous on the strip $\{z \in \mathbf{C}: \Im z \in[0,1 / 2]\}$ and holomorphic in the interior of this strip. For real $t(6.5)$ coincides with (6.4). Moreover

$$
\omega_{i / 2}(m)=\left(Q x \mid m Q^{-1} z\right) .
$$

It shows that $(\omega \otimes \mathrm{id}) W \in D\left(\tau_{i / 2}\right)$ and

$$
\tau_{i / 2}((\omega \otimes \mathrm{id}) W)=\left(\omega_{i / 2} \otimes \mathrm{id}\right) W
$$

Let $u, y \in H$. Then

$$
\left(u\left|\tau_{i / 2}((\omega \otimes \mathrm{id}) W)\right| y\right)=\left(u\left|\left(\omega_{i / 2} \otimes \mathrm{id}\right) W\right| y\right)=\left(Q x \otimes u|W| Q^{-1} z \otimes y\right) .
$$


Inserting in (1.7) $Q x$ and $Q^{-1} z$ instead of $x$ and $z$ respectively we get:

$$
\left(Q x \otimes u|W| Q^{-1} z \otimes y\right)=(\bar{z} \otimes u|\widetilde{W}| \bar{x} \otimes y)=\left(u\left|\left(\omega^{\top} \otimes \mathrm{id}\right) \widetilde{W}\right| y\right) .
$$

Combining the last two relations we obtain (6.2).

Ad. 3. We shall use a regularization operator. Let $n$ be a natural number. For any $a \in A$ we set

$$
R_{n}(a)=\frac{n}{\sqrt{\pi}} \int_{\mathbf{R}} e^{-n^{2} t^{2}} \tau_{t / 2}(a) d t .
$$

We know that $\tau_{t}(a)$ depends continuously on $t$. Therefore $\left\|R_{n}(a)-a\right\| \rightarrow 0$, when $n \rightarrow \infty$. Taking into account (6.3) one can easily show, that $R_{n} \mathcal{A} \subset \mathcal{A}$. One should notice that $\left\|R_{n}(a)\right\| \leq\|a\|$. Moreover $R_{n}(a) \in D\left(\tau_{i / 2}\right)$ and $\left\|\tau_{i / 2}\left(R_{n}(a)\right)\right\| \leq e^{n^{2}}\|a\|$. Indeed $\tau_{i / 2} R_{n}=\frac{n}{\sqrt{\pi}} \int \exp \left[-n^{2}(t-i)^{2}\right] \tau_{t / 2} d t,\left\|\tau_{i / 2} R_{n}\right\| \leq \frac{n}{\sqrt{\pi}} \int\left|\exp \left[-n^{2}(t-i)^{2}\right]\right| d t$ and the estimate follows.

Let $a \in D\left(\tau_{i / 2}\right), b=\tau_{i / 2}(a)$ and $\epsilon>0$. Then for sufficiently large $n$ we have

$$
\left\|R_{n}(a)-a\right\| \leq \frac{\epsilon}{2}, \quad\left\|R_{n}(b)-b\right\| \leq \frac{\epsilon}{2} .
$$

Clearly $\tau_{i / 2} R_{n}(a)=R_{n}(b)$. We know that $\mathcal{A}$ is dense in $A$. Let $a_{\epsilon}$ be an element of $\mathcal{A}$ such that

$$
\left\|a_{\epsilon}-a\right\| \leq \frac{\epsilon}{2} e^{-n^{2}}
$$

Then $R_{n}\left(a_{\epsilon}\right) \in \mathcal{A}$ and using the above estimates we have

$$
\begin{gathered}
\left\|R_{n}\left(a_{\epsilon}\right)-a\right\| \leq\left\|R_{n}\left(a_{\epsilon}-a\right)\right\|+\left\|R_{n}(a)-a\right\| \leq \frac{\epsilon}{2}\left(e^{-n^{2}}+1\right) \leq \epsilon, \\
\left\|\tau_{i / 2}\left(R_{n}\left(a_{\epsilon}\right)\right)-b\right\| \leq\left\|\tau_{i / 2} R_{n}\left(a_{\epsilon}-a\right)\right\|+\left\|R_{n}(b)-b\right\| \leq \epsilon .
\end{gathered}
$$

It shows that the closure of $\left.\tau_{i / 2}\right|_{\mathcal{A}}$ coincides with $\tau_{i / 2}$ and the statement follows.

Q.E.D.

\section{Unitary coinverse}

Let $K$ be a separable Hilbert space and $V \in B(K \otimes H)$ be a unitary adapted to $W$. For any $\varphi \in B(K)_{*}$ we denote by $\bar{\varphi} \in B(K)_{*}\left(\varphi^{\top} \in B(\bar{K})_{*}\right.$ respectively) the hermitian conjugate (the transposed respectively) functional:

$$
\begin{gathered}
\bar{\varphi}(m)=\overline{\varphi\left(m^{*}\right)} \\
\varphi^{\top}\left(m^{\top}\right)=\varphi(m)
\end{gathered}
$$

for any $m \in B(K)$. Let $x, y \in D\left(Q^{-1}\right), u, z \in D(Q)$ and $\varphi \in B(K)_{*}$. We shall use the notation introduced in Proposition 3.4. According to (3.7) and (3.6) we have:

$$
\begin{aligned}
\varphi\left((\mathrm{id} \otimes \mu) V(\mathrm{id} \otimes \nu)\left(V^{*}\right)\right) & =\varphi\left((\mathrm{id} \otimes \omega)\left(V^{*}\right)\right)=\omega\left((\varphi \otimes \mathrm{id})\left(V^{*}\right)\right) \\
& =\left(\bar{z} \otimes Q u\left|\widetilde{W}\left(\left[(\varphi \otimes \mathrm{id})\left(V^{*}\right)\right]^{\top} \otimes I\right) \widetilde{W}^{*}\right| \bar{x} \otimes Q^{-1} y\right) .
\end{aligned}
$$


Exchanging on the left hand side $x$ with $y$ and $u$ with $z$ (this operation changes $\mu$ into $\bar{\nu}$ and $\nu$ into $\bar{\mu}$ ) and replacing $\varphi$ by $\bar{\varphi}$ we obtain a number complex conjugate to the original one. Therefore

$$
\begin{aligned}
(\bar{z} \otimes Q u \mid \widetilde{W} & \left.\left(\left[(\varphi \otimes \mathrm{id})\left(V^{*}\right)\right]^{\top} \otimes I\right) \widetilde{W}^{*} \mid \bar{x} \otimes Q^{-1} y\right) \\
& =\overline{\left(\bar{u} \otimes Q z\left|\widetilde{W}\left([(\varphi \otimes \mathrm{id}) V]^{* \top} \otimes I\right) \widetilde{W}^{*}\right| \bar{y} \otimes Q^{-1} x\right)} \\
& =\left(\bar{y} \otimes Q^{-1} x\left|\widetilde{W}\left([(\varphi \otimes \mathrm{id}) V]^{\top} \otimes I\right) \widetilde{W}^{*}\right| \bar{u} \otimes Q z\right) .
\end{aligned}
$$

Let $R H S$ denotes the right hand side of (7.1). Taking into account (1.6) we have:

$$
\begin{aligned}
R H S & =\left(\bar{y} \otimes Q^{-1} x\left|\widetilde{W}\left([(\varphi \otimes \mathrm{id}) V]^{\top} \otimes I\right) \widetilde{W}^{*}\left(\left\{Q^{\top}\right\}^{-1} \otimes Q\right)\right| \overline{Q u} \otimes z\right) \\
& =\left(\bar{y} \otimes Q^{-1} x\left|\widetilde{W}\left([(\varphi \otimes \mathrm{id}) V]^{\top} \otimes I\right)\left(\left\{Q^{\top}\right\}^{-1} \otimes Q\right) \widetilde{W}^{*}\right| \overline{Q u} \otimes z\right)
\end{aligned}
$$

Using (1.16) one can easily show, that

$$
\left[\left(\varphi^{\top} \otimes \mathrm{id}\right) \tilde{V}\right] Q^{-1} \subset Q^{-1}[(\varphi \otimes \mathrm{id}) V]
$$

Therefore (passing to transposed operators inverts the inclusion)

$$
\begin{gathered}
\left\{Q^{\top}\right\}^{-1}\left[\left(\varphi^{\top} \otimes \mathrm{id}\right) \tilde{V}\right]^{\top} \supset[(\varphi \otimes \mathrm{id}) V]^{\top}\left\{Q^{\top}\right\}^{-1} \\
\left(\left\{Q^{\top}\right\}^{-1} \otimes Q\right)\left(\left[\left(\varphi^{\top} \otimes \mathrm{id}\right) \tilde{V}\right]^{\top} \otimes I\right) \supset\left([(\varphi \otimes \mathrm{id}) V]^{\top} \otimes I\right)\left(\left\{Q^{\top}\right\}^{-1} \otimes Q\right)
\end{gathered}
$$

and

$$
R H S=\left(\bar{y} \otimes Q^{-1} x\left|\widetilde{W}\left(\left\{Q^{\top}\right\}^{-1} \otimes Q\right)\left(\left[\left(\varphi^{\top} \otimes \mathrm{id}\right) \widetilde{V}\right]^{\top} \otimes I\right) \widetilde{W}^{*}\right| \overline{Q u} \otimes z\right) .
$$

Using once more (1.6) we get

$$
\begin{aligned}
R H S & =\left(\bar{y} \otimes Q^{-1} x\left|\left(\left\{Q^{\top}\right\}^{-1} \otimes Q\right) \widetilde{W}\left(\left[\left(\varphi^{\top} \otimes \mathrm{id}\right) \widetilde{V}\right]^{\top} \otimes I\right) \widetilde{W}^{*}\right| \overline{Q u} \otimes z\right) \\
& =\left(\overline{Q^{-1} y} \otimes x\left|\widetilde{W}\left(\left[\left(\varphi^{\top} \otimes \mathrm{id}\right) \widetilde{V}\right]^{\top} \otimes I\right) \widetilde{W}^{*}\right| \overline{Q u} \otimes z\right) .
\end{aligned}
$$

Formula (7.1) takes now the following form:

$$
\begin{aligned}
(\bar{z} \otimes Q u \mid & \widetilde{W} \\
= & \left.\left(\left[(\varphi \otimes \mathrm{id})\left(V^{*}\right)\right]^{\top} \otimes I\right) \widetilde{W}^{*} \mid \bar{x} \otimes Q^{-1} y\right) \\
& =\left(\overline{Q^{-1} y} \otimes x\left|\widetilde{W}\left(\left[\left(\varphi^{\top} \otimes \mathrm{id}\right) \widetilde{V}\right]^{\top} \otimes I\right) \widetilde{W}^{*}\right| \overline{Q u} \otimes z\right)
\end{aligned}
$$

and using the antiunitary operator (3.2) we get

$$
\left(J \Phi\left|\widetilde{W}\left(\left[(\varphi \otimes \mathrm{id})\left(V^{*}\right)\right]^{\top} \otimes I\right) \widetilde{W}^{*}\right| J \Psi\right)=\left(\Psi\left|\widetilde{W}\left(\left[\left(\varphi^{\top} \otimes \mathrm{id}\right) \widetilde{V}\right]^{\top} \otimes I\right) \widetilde{W}^{*}\right| \Phi\right),
$$


where $\Phi=\overline{Q u} \otimes z$ and $\Psi=\overline{Q^{-1} y} \otimes x$ run over a linearly dense subsets of $\bar{H} \otimes H$. Therefore the above formula holds for all $\Phi, \Psi \in \bar{H} \otimes H$. Remembering that $J$ is an antiunitary involution we get

$$
J \widetilde{W}\left(\left[(\varphi \otimes \mathrm{id})\left(V^{*}\right)\right]^{\top} \otimes I\right)^{*} \widetilde{W}^{*} J=\widetilde{W}\left(\left[\left(\varphi^{\top} \otimes \mathrm{id}\right) \widetilde{V}\right]^{\top} \otimes I\right) \widetilde{W}^{*} .
$$

One can easily verify that $J\left(a^{\top} \otimes I\right) J=I \otimes a^{*}$ for any $a \in B(H)$. Therefore

$$
J \widetilde{W} J\left(I \otimes\left[(\varphi \otimes \mathrm{id})\left(V^{*}\right)\right]\right) J \widetilde{W}^{*} J=\widetilde{W} J\left(I \otimes\left[\left(\varphi^{\top} \otimes \mathrm{id}\right) \widetilde{V}\right]^{*}\right) J \widetilde{W}^{*}
$$

and finally

$$
\widetilde{J}\left(I \otimes\left[(\varphi \otimes \mathrm{id})\left(V^{*}\right)\right]\right) \widetilde{J}=I \otimes\left[\left(\varphi^{\top} \otimes \mathrm{id}\right) \widetilde{V}\right]^{*},
$$

where $\widetilde{J}$ is an antiunitary involution acting on $\bar{H} \otimes H$ introduced by

$$
\widetilde{J}=J \widetilde{W}^{*} J \widetilde{W} J
$$

Assume for the moment that $K=H$ and $V=W$. In this special case (7.3) takes the form

$$
\widetilde{J}\left(I \otimes\left[(\omega \otimes \mathrm{id})\left(W^{*}\right)\right]\right) \widetilde{J}=I \otimes\left[\left(\omega^{\top} \otimes \mathrm{id}\right) \widetilde{W}\right]^{*},
$$

where $\omega \in B(H)_{*}$ and $\omega^{\top} \in B(\bar{H})_{*}$ is related to $\omega$ by the formula: $\omega^{\top}\left(a^{\top}\right)=\omega(a)$ for all $a \in B(H)$. We already know (cf Remark following Proposition 6.1) that ( $\omega^{\top} \otimes$ id) $\widetilde{W} \in$ $A$. Remembering $(\mathrm{cf}(1.9))$ that $(\omega \otimes \mathrm{id})\left(W^{*}\right)$ runs over a dense subset of $A$ we obtain Statement 1 of the following Proposition:

\section{Proposition 7.1}

1. For any $a \in A$ there exists $a^{R} \in A$ such that

$$
\widetilde{J}(I \otimes a) \widetilde{J}=I \otimes\left[a^{R}\right]^{*} .
$$

2. The mapping

$$
A \ni a \longmapsto a^{R} \in A
$$

is an involutive antiautomorphism acting on the $C^{*}$-algebra $A$.

3. $R$ commutes with the action of the scaling group $\left(\tau_{t}\right)_{t \in \mathbf{R}}$.

Proof: Statement 1 is already proven.

Ad 2. It follows immediately from (7.6), that the correspondence $a \mapsto\left[a^{R}\right]^{*}$ is antilinear, multiplicative isometric and ultraweakly continuous. Consequently (7.7) is linear, antimultiplicative isometric and normal. Applying the hermitian conjugation to the both sides of (7.6) and comparing the result with the original formula we get $a^{* R *}=a^{R}$. It shows that (7.7) commutes with the hermitian conjugation. On the other hand the antiunitary (7.4) is involutive. Therefore $a^{R * R *}=a$ and $a^{R R}=a$ for all $a \in A$. So (7.7) is involutive and consequently its range coincides with $A$. 
Ad 3. Using (1.6) one can easily show that antiunitary (7.4) anticommutes with $Q^{\top} \otimes Q^{-1}: \quad \widetilde{J}\left(Q^{\top} \otimes Q^{-1}\right) \widetilde{J}=\left(Q^{\top} \otimes Q^{-1}\right)^{-1}$. Therefore for any real $t$ we have $\widetilde{J}\left(Q^{\top} \otimes Q^{-1}\right)^{i t} \widetilde{J}=\left(Q^{\top} \otimes Q^{-1}\right)^{i t}$ and the Statement follows.

Q.E.D.

Let $\omega \in B(H)_{*}$. Formula (7.5) shows that $\left[(\omega \otimes \mathrm{id}) W^{*}\right]^{R}=\left(\omega^{\top} \otimes \mathrm{id}\right) \widetilde{W}$. Therefore

$$
(\omega \otimes \mathrm{id}) W^{*}=\left[\left(\omega^{\top} \otimes \mathrm{id}\right) \widetilde{W}\right]^{R} .
$$

One can easily see that this formula is equivalent to (1.14).

To complete the proof of Theorem 1.5 we have to demonstrate Statement 4 . To this end we use the polar decomposition (1.13) as definition of $\kappa$ : for any $a \in D\left(\tau_{i / 2}\right)$ we set

$$
\kappa(a)=\tau_{i / 2}(a)^{R}
$$

Then (cf Proposition 6.1.3) $\left\{(\omega \otimes \mathrm{id}) W: \omega \in B(H)_{*}\right\}$ is a core of $\kappa$ and (1.12) holds. Indeed, using (6.2) and (7.8), we get:

$$
\kappa((\omega \otimes \mathrm{id}) W)=\left[\left(\omega^{\top} \otimes \mathrm{id}\right) \widetilde{W}\right]^{R}=(\omega \otimes \mathrm{id}) W^{*} .
$$

The algebraic properties of $\kappa$ described in Statement 4 follows immediately from the polar decomposition (1.13). Theorem 1.5 is proved.

In the similar way we can complete the proof of Theorem 1.6. By the general theory, the action of $R$ and $\tau_{i / 2}$ admits canonical extension to $\mathrm{M}(\mathrm{A})$. Let $\varphi \in B(K)_{*}$. Formula (7.3) shows that

$$
\left[(\varphi \otimes \mathrm{id}) V^{*}\right]^{R}=\left(\varphi^{\top} \otimes \mathrm{id}\right) \tilde{V}
$$

and Statement 5 follows. Moreover the above equality proves that $\left(\varphi^{\top} \otimes \mathrm{id}\right) \tilde{V} \in M(A)$. The relation (7.2) shows now, that $(\varphi \otimes \mathrm{id}) V \in D\left(\tau_{i / 2}\right)$ and

$$
\tau_{i / 2}((\varphi \otimes \mathrm{id}) V)=\left(\varphi^{\top} \otimes \mathrm{id}\right) \widetilde{V}
$$

Combining the last two formulae we obtain Statement 4. Theorem 1.6 is proved.

\section{References}

[1] S. Baaj: Représentation regulière du groupe quantique $E_{\mu}(2)$. C.R. Acad. Sci. 314 (1992) $1021-1026$.

[2] S. Baaj: Représentation regulière du groupe quantique des deplacements de Woronowicz. Preprint, Département de Mathématiques et d'Informatique, Université d'Orléans 1994.

[3] S. Baaj and G. Skandalis: Unitaires multiplicative et dualité pour les produits croisés de $C^{*}$-algèbres. Ann. scient. Éc. Norm. Sup., $4^{e}$ série, t. 26, 1993, pp 425 488. 
[4] T. Masuda, Y. Nakagami and S.L. Woronowicz: in preparation

[5] G. Skandalis: Operator algebras and duality. Proceedings of the International Congress of Mathematicians, Kyoto, Japan 1990, Springer - Verlag.

[6] N. Tatsuuma: A duality theorem for locally compact groups. J. of Math. of Kyoto Univ., vol 6, 1967 pp $187-293$.

[7] D. Wichmann: Approximate identities and factorization in Banach modules. Lecture Notes in Mathematics No 768. Springer Verlag 1979.

[8] S.L. Woronowicz: C*-algebras generated by unbounded elements. Reviews in Mathematical Physics, Vol. 7, No.3 (1995) 481 - 521

[9] L. Zsido: On the characterization of the analytic generator of *automorphism groups in Operator algebras and applications (Kingstone Ontario 1980) Proc. Sympos. Pure Math. 38 AMS Providence R.I. 1982, pp 381 - 383. 